\title{
Akt3 inhibits adipogenesis and protects from diet-induced obesity via WNK1/SGK1 signaling
}

\author{
Liang Ding, ${ }^{1}$ Lifang Zhang, ${ }^{1}$ Sudipta Biswas, ${ }^{1}$ Rebecca C. Schugar, ${ }^{2}$ J. Mark Brown, ${ }^{2}$ Tatiana Byzova, ${ }^{1}$ \\ and Eugene Podrez ${ }^{1}$
}

'Department of Molecular Cardiology and 'Department of Cellular and Molecular Medicine, Cleveland Clinic, Cleveland, Ohio, USA.

Three Akt isoforms, encoded by 3 separate genes, are expressed in mammals. While the roles of Akt1 and Akt2 in metabolism are well established, it is not yet known whether Akt3 plays a role in metabolic diseases. We now report that Akt3 protects mice from high-fat diet-induced obesity by suppressing an alternative pathway of adipogenesis via with no lysine protein kinase-1 (WNK1) and serum/glucocorticoid-inducible kinase 1 (SGK1). We demonstrate that Akt3 specifically phosphorylates WNK1 at T58 and promotes its degradation via the ubiquitin-proteasome pathway. A lack of Akt3 in adipocytes increases the WNK1 protein level, leading to activation of SCK1. SCK1, in turn, promotes adipogenesis by phosphorylating and inhibiting transcription factor FOXO1 and, subsequently, activating the transcription of PPAR $\gamma$ in adipocytes. Akt3-deficient mice have an increased number of adipocytes and, when fed a high-fat diet, display increased weight gain, white adipose tissue expansion, and impaired glucose homeostasis. Pharmacological blockade of SCK1 in high-fat diet-fed Akt3-deficient mice suppressed adipogenesis, prevented excessive weight gain and adiposity, and ameliorated metabolic parameters. Thus, Akt3/WNK1/SGK1 represents a potentially novel signaling pathway controlling the development of obesity.

Conflict of interest: The authors have declared that no conflict of interest exists.

Submitted: June 13, 2017

Accepted: October 17, 2017

Published: November 16, 2017

Reference information: JCI Insight. 2017;2(22):e95687. https://doi.org/10.1172/jci. insight.95687.

\section{Introduction}

Adipocyte hyperplasia plays an important role in the development of obesity and its complications, such as insulin resistance. It plays a critical role in childhood-onset obesity and strongly predisposes for later-in-life obesity (1). To date, the mechanisms regulating adipogenesis in vivo are still incompletely understood. In vitro studies have demonstrated a transcriptional cascade that is required for white adipogenesis, involving the sequential induction of a variety of transcription factors (2), including FOXO1, several members of the $\mathrm{C} / \mathrm{EBP}$ family, and PPAR $\gamma$ at the terminal differentiation stage $(2,3)$. FOXO1 is an essential negative regulator of adipogenesis (4), and it is primarily controlled through phosphorylation/acetylation on multiple residues by enzymes, including Akt kinase (5).

Akt regulates numerous cellular processes, including cell growth, differentiation, proliferation, apoptosis, and glucose metabolism (5). Three Akt isoforms, encoded by 3 separate genes, are expressed in mammals. They are highly homologous and display similar substrate specificity. Phenotypic analysis of the individual Akt isoform deletions and combined deletions in the mouse suggest compensatory and complementary roles of isoforms (5). However, the 3 isoforms also have distinct, even opposite, functions - even at the cellular level $(6,7)$. All 3 isoforms of Akt are expressed in adipocytes (8). The roles of Akt1 and Akt2 in adipocyte function have studied both in vivo and in vitro. Akt1 in primary mouse embryonic fibroblasts (MEF) has been shown to promote adipocyte differentiation (9). Similarly, Akt2 promotes preadipocyte proliferation and adipogenesis, and it regulates adipocyte apoptosis (10-12). Akt2 also plays a key role in the activation of adipocyte precursor cells in response to a high-fat diet (HFD) (13). Adipocyte-specific deletion of Akt1/2 causes lipodystrophy, insulin resistance, fatty liver, and enlarged islets of Langerhans (14). FOXO1 phosphorylation by Akt1 is considered to be a major mechanism of Akt1 contribution to adipogenesis (9), and transduction of insulin induced signaling at the cellular membrane, a major contribution of Akt2 (15). In contrast to Akt1 and Akt2, the role of Akt3 in metabolism, and specifically in adipocyte function, has not yet been described. 
Another kinase that can phosphorylate FOXO1 and regulate FOXO1 function is SGK1 (4). SGK1 is a serine/threonine protein kinase that acts downstream of phosphoinositide 3-kinase and regulates various cellular functions (16). Several studies have indicated that SGK1 might contribute to the development of fat tissue. A SGK1 gene variant predisposes to hypertension, stroke, obesity, and type 2 diabetes (17). Recent in vitro studies have suggested that, similar to Akt1, SGK1 can contribute to obesity by phosphorylating and inhibiting FOXO1 in preadipocytes (4). However, conditions that may lead to sustained upregulation of SGK1 activity in vivo and pathophysiological consequences of such upregulation in vivo are not known. Interestingly, SGK1 has a complex and poorly understood relationship with Akt and another serine/threonine protein kinase: WNK1. WNK1 forms a complex with SGK1 and promotes SGK1 activation via an unknown mechanism (18). At the same time, WNK1 can be phosphorylated by both Akt and SGK1; however, the role of this phosphorylation in WNK1 function is unclear.

Here, we used multiple in vivo and in vitro approaches to document the existence and critical role of the Akt3/WNK1/SGK1 signaling pathway in adipocytes. We demonstrated that dysregulation of this pathway increases adipogenesis, predisposing mice to obesity. Mechanistically, the Akt3 activity in preadipocytes suppresses adipogenesis via phosphorylation of WNK1, reducing protein stability of WNK1, leading to downregulation of SGK1 activity and SGK1-mediated inhibition of FOXO1. We demonstrated that Akt3-deficient mice have an increased number of adipocytes, and when fed an HFD, display increased weight gain and fat expansion. We further demonstrated that the small molecule inhibitor of SGK1 prevented diet-induced obesity and ameliorated metabolic parameters in Akt3-deficient mice by inhibiting adipogenesis.

\section{Results}

Deficiency of Akt3 in preadipocytes promotes adipogenesis. We tested the role of 3 isoforms of Akt kinase in adipocyte differentiation using $\mathrm{MEF}$ isolated from $\mathrm{Akt1}^{-/-}, \mathrm{Akt}^{-/-}$, and $\mathrm{Ak}^{-/-}$mice. Confluent $\mathrm{MEF}$ were cultured in differentiation media and adipogenesis assed by Oil red $\mathrm{O}$ staining (Figure 1A). We observed reduced adipogenesis in $A k t 1^{-/-} \mathrm{MEF}$, as compared with WT MEF (Figure 1A). Adipogenesis in $A k t 2^{-1-}$ MEF was similar to WT MEF (Figure 1A). Surprisingly, we observed significantly enhanced adipogenesis in $\mathrm{Akt}^{3^{--}} \mathrm{MEF}$ (Figure 1A). The overexpression of Akt3 in $A k t 3^{-1-}$ MEF rescued the phenotype (Figure 1B), supporting the specific role of Akt3 in the regulation of adipogenesis. These results were reproduced using the siRNA-induced silencing of Akt3 or Akt1 expression in 3T3 L1 preadipocytes (Figure 1C, upper panel, and Figure 1D). Overexpression of the Akt3 mutant possessing enhanced kinase activity $\left(\mathrm{Akt}^{\mathrm{nm} 3350}\right)$ (19) significantly inhibited adipogenesis, while overexpression of WT Akt3 modestly inhibited adipogenesis in 3T3 L1 preadipocytes (Figure 1C, lower panel, and Figure 1D), further implicating Akt3 activity in adipogenesis. The inhibition of adipocyte differentiation by Akt3 was also reproduced in human s.c. preadipocytes with Akt3 knockdown induced by specific siRNA (Figure 1E). To confirm the effect of Akt3 on adipocyte differentiation at protein expression level, we assessed the protein levels of PPAR $\gamma$ and $\mathrm{C} / \mathrm{EBP} \alpha, 2$ critical adipogenic transcription factors (20) and markers of adipocyte differentiation, fatty acid binding protein 4 (FABP4), and fatty acid synthase (FAS, also known as adipocyte protein 2 [aP2]). We observed enhanced protein level of PPAR $\gamma, \mathrm{C} / \mathrm{EBP} \alpha$, and FABP4 in Akt3-deficient MEF-differentiated adipocytes as compared with WT cells (Figure 1F). The protein level of FAS, an enzyme that plays a central role in fatty acid and triglyceride synthesis, was significantly increased in $\mathrm{Akt}^{-1-} \mathrm{MEF}$-differentiated adipocytes (Figure $1 \mathrm{~F}$ ).

As Akt activation has been reported to inhibit lipolysis (21), we tested the effect of Akt3 deficiency on the expression/activity of lipolytic enzymes. We found a moderate increase in the expression of adipose triglyceride lipase (ATGL) and monoacylglycerol lipase (MGL) (Figure 1F) in $A k t 3^{-1-}$ MEF. While we did not find changes in the expression of hormone-sensitive lipase (HSL), we observed moderately enhanced phosphorylation of HSL at Ser563 in $A k t 3^{-1-} \mathrm{MEF}$-differentiated adipocytes, indicating the activation of HSL in these cells. A strong increase (6-fold) in the lipogenic enzyme expression and a relatively moderate increase (1.5- to 2-fold) in lipolytic enzymes suggest that the lipogenesis prevails in $A k t 3^{-1-} \mathrm{MEF}$-differentiated adipocytes, likely contributing to an increased triglyceride content in these cells (Figure 1G). Taken together, these results demonstrate that, in contrast with other Akt isoforms, Akt3 activity inhibits adipogenesis.

FOXO1 is a pivotal negative regulator of adipogenesis (4) via repression of transcription of $\operatorname{PPAR} \gamma(22)$. It is also a critical positive regulator of adiponectin gene transcription (23) in adipocytes. Adiponectin production and circulating levels reflect FOXO1 activity (23). We observed that levels of 
A



C Control


E

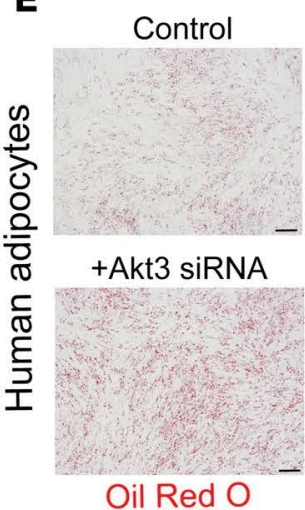



Akt1

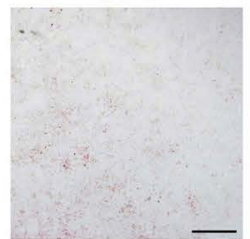

Akt3 ${ }^{\text {nmf350 }}$

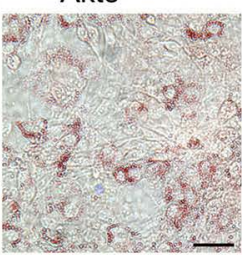

Akt2-r

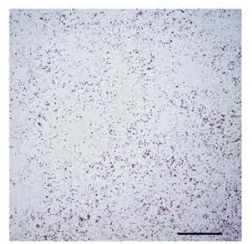

D


B


Akt1 siRNA

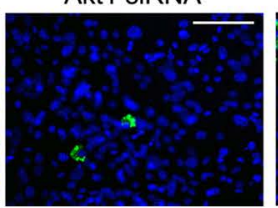

Akt3 ${ }^{\text {nm+350 }}$
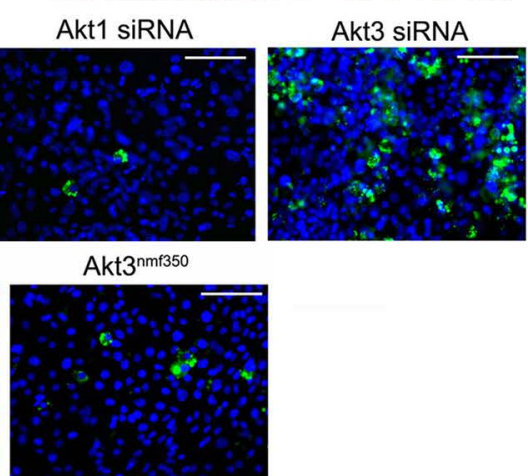

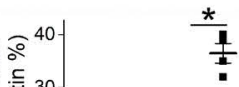

G

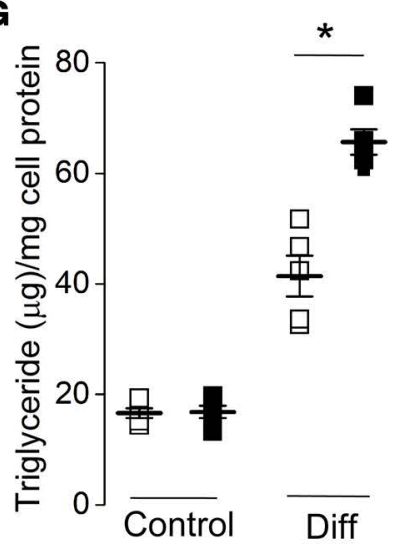

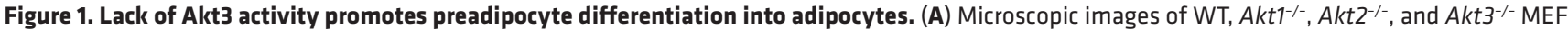
at day 8 after induction of adipogenesis. Scale bars: $500 \mu \mathrm{m} . n=5$. (B) Oil red 0 staining of $A k t 3^{-1-}$ MEF-differentiated adipocytes after treatment with plasmid encoding Akt3 gene. Scale bar: $200 \mu \mathrm{m} . n=3$. (C) Oil red 0 staining of 3T3 differentiated adipocytes after treatment with Akt1-specific siRNA, Akt3-specific siRNA, WT Akt3 plasmid, or mutant Akt3 (Akt3 ${ }^{n+350}$ ) plasmid. 3T3-L1 cells were transfected with indicated siRNA or plasmids for 24 hours before addition of differentiation medium. Scale bar: $50 \mu \mathrm{m} . n=5$. (D) BODIPY 493/503 staining of 3 T3 differentiated adipocytes after treatment with Akt1-specific siRNA, Akt3-specific siRNA, WT Akt3 plasmid, or mutant Akt3 (Akt3 ${ }^{\text {nmf350) }}$ plasmid. Scale bar: $100 \mu m$. $n=5$. (E) Adipogenesis in human s.c. preadipocytes treated with Akt3 siRNA. Scale bar: $200 \mu \mathrm{m} . n=3$. (F) Western blot analysis of FABP4, C/EBP $\alpha$, PPAR $\gamma$, FAS, HSL, ATGL, MGL, and phosphorylation of HSL (Ser563) in WT and Akt3-l- MEF-differentiated adipocytes. Right graphs show densitometric quantification. $n=4$. (C) The content of triglyceride in WT and $A k t 3^{-/-}$MEF before (control) or after (Diff) 1-week incubation in adipogenic media containing dexamethasone, insulin, and IBMX. $n=$ 5. Data represent means \pm SEM. ${ }^{*} P<0.05$ by 2 -tailed Student's $t$ test.

adiponectin in circulation of $A k t 3^{---}$mice were reduced by $45 \%$ as compared with WT mice (Figure 2A). Adiponectin production by $A k t 3^{---} \mathrm{MEF}$ was also reduced as compared with WT and $A k t 1^{-1-}$ adipocytes (Figure 2B). These results suggest reduced activity of FOXO1 in adipocytes, which in turn may explain the increased adipogenesis in $A k t 3^{-1-} \mathrm{MEF}$.

The level of FOXO1 in the nuclei of adipocytes is regulated by phosphorylation (9). Upon phosphorylation, FOXO1 is exported from the nucleus and degraded via a ubiquitin-proteasome pathway, leading to increased expression of PPAR $\gamma$ and other adipogenic genes (24). In agreement with reduced levels of adiponectin and increased adipogenesis, we observed enhanced phosphorylation and decreased FOXO1 
A

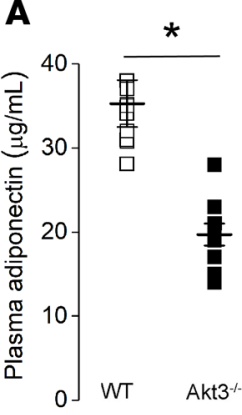

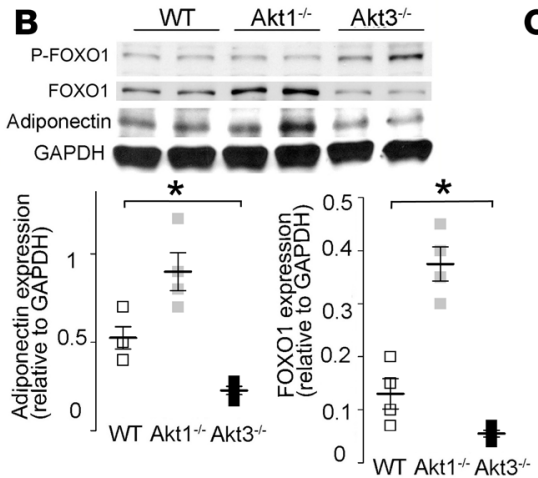

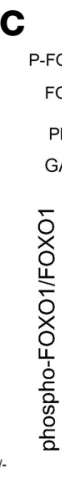

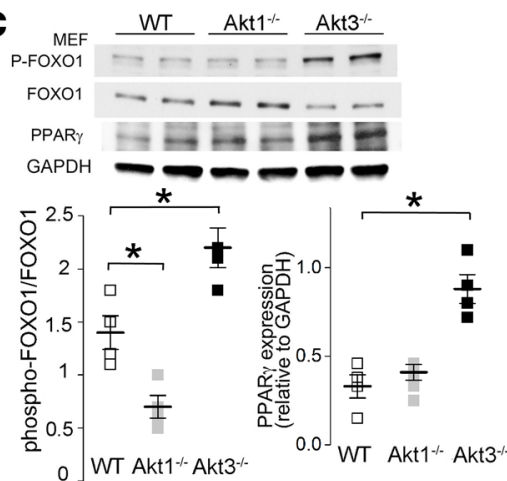

E ctl Akt3i Akt1i

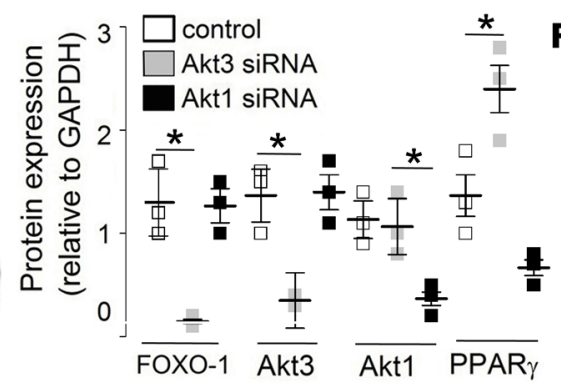

$F$

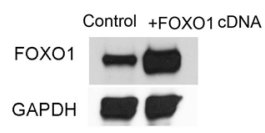

control

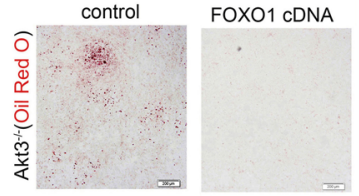

$\mathbf{H}$
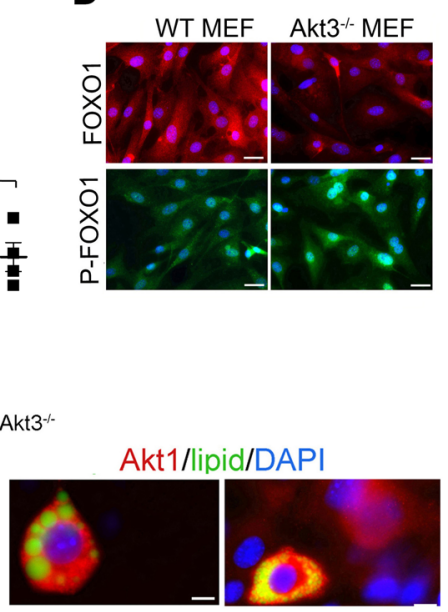

D

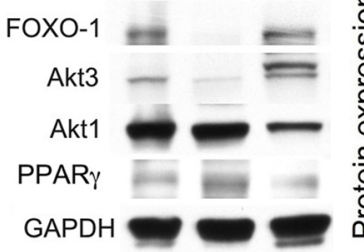

FOXO-1 Akt3
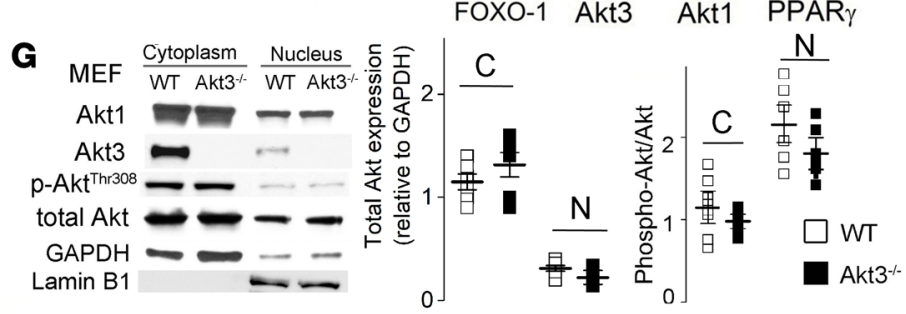
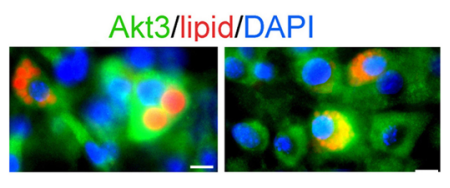

I


Figure 2. Akt3 regulates adipogenesis via a FoX01 pathway. (A) Plasma adiponectin levels in 20-week-old WT and $A k t 3^{-/-}$mice on a chow diet $(n=10,5$ male, 5 female) were assessed by a mouse adiponectin ELISA kit. (B) Western blot analyses of FOX01, phospho-FOXO1, and PPAR $\gamma$ expression in mouse embryonic fibroblasts (MEF) from WT, $A k t 1^{-1-}$, and $A k t 3^{-1-}$ MEF. $n=4$. Lower graphs show densitometric quantification. (C) Western blot analysis of FOXO1, phospho-FOXO1, and adiponectin expression in MEF differentiated adipocytes. $n=4$. Lower graphs show densitometric quantification. (D) Immunofluorescent staining of WT and Akt3-/- MEF using anti-FOXO1 or anti-phospho-FOXO1 (Ser256) antibody. Scale bar: $25 \mu \mathrm{m}$. $n=5$. (E) Expression of FOXO1, PPAR $\gamma, A k t 1$, and Akt3 in $3 T 3$ differentiated adipocytes after treatment with indicated siRNA. $n=3$. Right graph shows densitometric quantification. (F) Oil red $O$ staining of ferentiated adipocytes after treatment with plasmid encoding FOXO1 gene. Scale bar: $200 \mu \mathrm{m}$. $n=3$. (C) The cytoplasmic and nuclear expression of Akt1, Akt3, p-Akt (thr308), and total Akt in WT and Akt3-/- MEF. Lamin B1 was used as loading controls. Right panel shows subcellular localization of Akt1 and Akt3 in WT MEF. $n=3$. C, cytoplasm; N, nucleus. (H) Subcellular localization of Akt1 and Akt3 in 3T3 differentiated adipocytes. Lipid was stained with BODIPY 493/503 (green) or HCS LipidTOX Red Neutral Lipid Stain (red). DAPI (blue) was used as the nuclear marker. Scale bar: 10 $\mu \mathrm{m} . n=4$. (I) Expression of Akt1, Akt2, Akt3, pan-Akt, and phospho-Akt (Ser473) in WT, Akt1 ${ }^{-1-}$, and Akt3-/- MEF. Graphs on the right show densitometric quantification. $n=4$. Right graph shows densitometric quantification. Data represent means $\pm \mathrm{SEM} .{ }^{*} P<0.05$ by 2 -tailed Student's $t$ test.

levels and, correspondingly, an increased PPAR $\gamma$ level in $A k t 3^{--}$MEF, as compared with WT MEF (Figure 2C). In contrast, $A k t 1^{---}$MEF had increased FOXO1 levels and a reduced phospho-FOXO1/FOXO1 ratio, reflecting the major role of Akt1 in phosphorylation of FOXO1 in these cells (Figure 2C). Immunofluorescent staining revealed increased phospho-FOXO1 in nuclei and reduced FOXO1 in cytoplasm of $A k t 3^{-1-}$ MEF (Figure 2D). These observations were further confirmed using 3T3-L1 preadipocytes with an expression of either Akt3 or Akt1 silenced by specific siRNA (Figure 2E). We also assessed FOXO1 and phospho-FOXO1 levels in MEF-derived differentiated adipocytes. FOXO1 levels were reduced and phospho-FOXO1 levels were increased in $A k t 3^{-/-}$MEF adipocytes as compared with WT and $A k t 1^{-/-}$MEF adipocytes (Figure 2B). The overexpression of FOXO1 in $A k t 3^{-1-} \mathrm{MEF}$ suppressed adipogenesis (Figure $2 \mathrm{~F}$ ), supporting the specific role of FOXO1 in the regulation of adipogenesis in these cells. Taken together, these results show that, in contrast with 2 other Akt isoforms, Akt3 isoform is antiadipogenic. These results also demonstrate that the effect of Akt3 is mediated by regulating FOXO1 phosphorylation. Interestingly, while differential intracellular localization is commonly used to explain nonoverlapping functions of Akt isoforms expressed in the same cell, immunocytochemistry analysis revealed a similar mixed cytoplasmic and nuclear presence of Akt1 and Akt3 in both 3T3-L1 preadipocytes and in MEF (Figure 2, G and H). 

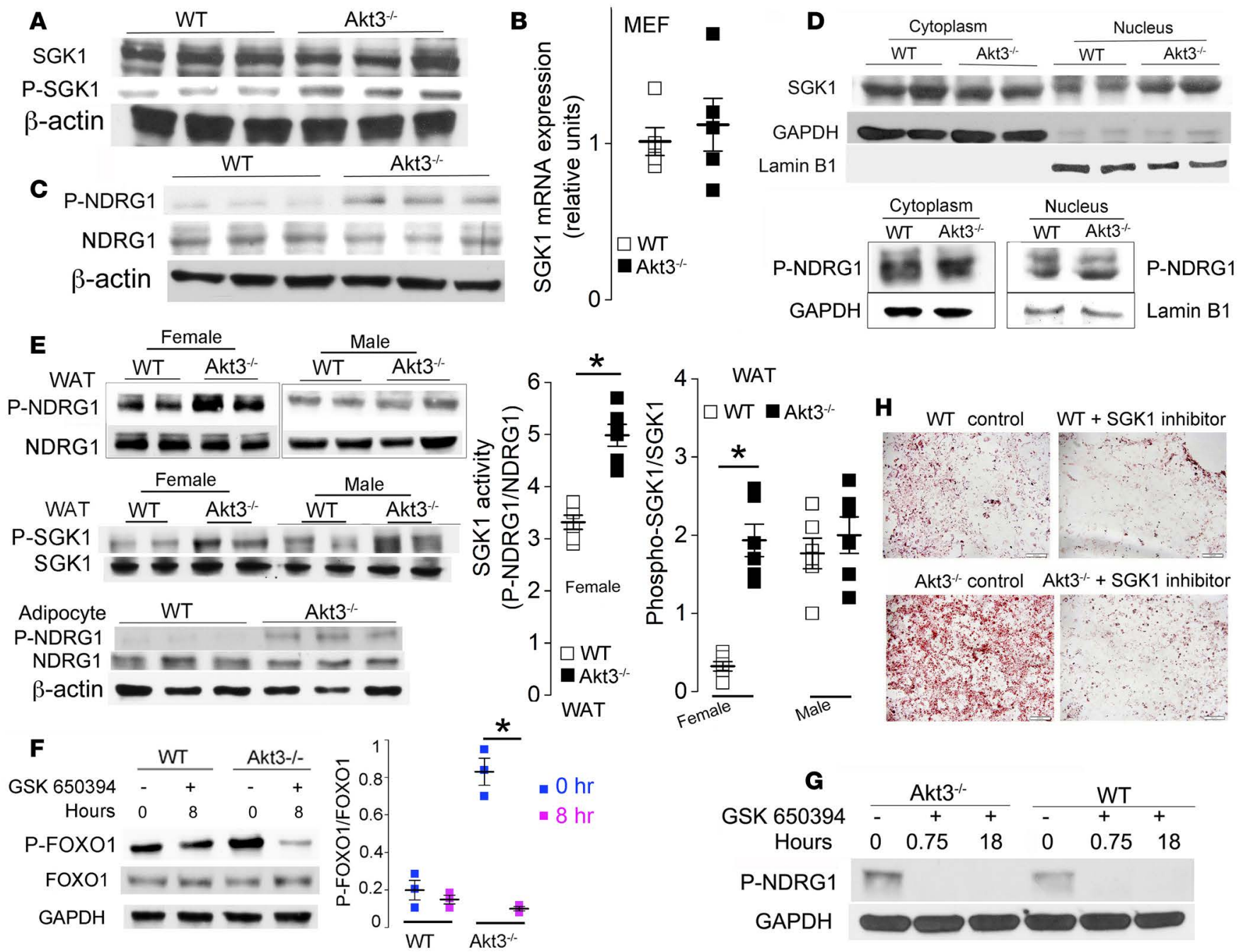

Figure 3. Akt3 regulates adipogenesis via a SGK1/F0X01 pathway. (A) Western blot analysis of SGK1 and phospho-SGK1 in WT and Akt3-/- MEF ( $n=3$ ).


$(n=3)$. (D) The cytoplasmic and nuclear expression of SGK1 and phospho-NDRG1 in WT and Akt3 ${ }^{-1-}$ MEF. GAPDH and Lamin B1 as loading controls. $n=4$. (E) Western blot analysis of SGK1, phospho-SGK1, NDRG1, and phospho-NDRG1 in WAT of female/male WT and Akt3 $3^{-/-}$mice fed a chow diet ( $n=8$, 4 females, 4 males). Graphs show SGK1 activity (phosphorylation of NDRG1) in adipocytes isolated from WAT of female mice on a chow diet $(n=6)$. (F) The expression of phospho-FOX01 and FOX01 in WT and Akt3-/- MEF in the presence of SGK1 inhibitor (GSK650394) at different time points. $n=3$. Right graph shows densitometric quantification of phospho-FOXO1 levels relative to total FOXO1 levels. (G) Phosphorylation of NDRG1 in WT and $A k t 3^{-1-}$ MEF in the presence of GSK650394 at different time points. $n=3$. (H) The differentiation of WT and Akt3 ${ }^{-1-}$ MEF into adipocytes was substantially reduced in the presence of GSK650394. Scale bar $=100 \mu \mathrm{m} . n=5$. Data represent means \pm SEM. ${ }^{*} P<0.05$ by 2 -tailed Student's $t$ test.

As Akt1 is responsible for FOXO1 phosphorylation during adipocyte differentiation $(9,11)$ and Akt1 deficiency suppresses adipogenesis (Figure 1A), we tested whether Akt3 regulates Akt1 expression or activity in adipocytes. Deletion of Akt3 had no significant effect on total Akt level and Akt phosphorylation (Ser473) in MEF (Figure 2I). Furthermore, we found no increase in nuclear localization of Akt1 in $A k t 3^{-/-}$cells or nuclear phosphorylated Akt (Thr308) (Figure 2G), ruling out the role for Akt1 in downregulation of FOXO1 in $A k t 3^{---}$cells.

SGK1 is responsible for downregulation of FOXO1 in Akt3 ${ }^{-/-}$cells. FOXO1 can be phosphorylated by several kinases in vitro $(25,26)$. SGK1 is of particular interest, since it can phosphorylate FOXO1 at the same sites as Akt (27), and in vitro studies have suggested that it may play a role in adipocyte differentiation (25). Western blotting and quantitative PCR (qPCR) revealed similar SGK1 protein levels but increased phospho-SGK1 (Ser422) in $A k t 3^{-1-}$ MEF (Figure 3, A and B), suggesting increased SGK1 activity. Phosphorylation of NDRG1, a SGK1-specific phosphorylation substrate (28), was significantly increased in $A k t 3^{-1-}$ MEF, confirming enhanced SGK1 activity (Figure 3C). SGK1 level and kinase activity were specifi- 
A
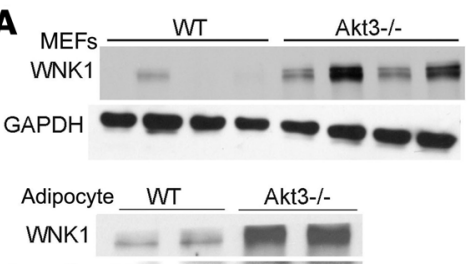



C



+Akt3 siRNA

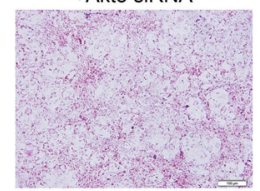

G
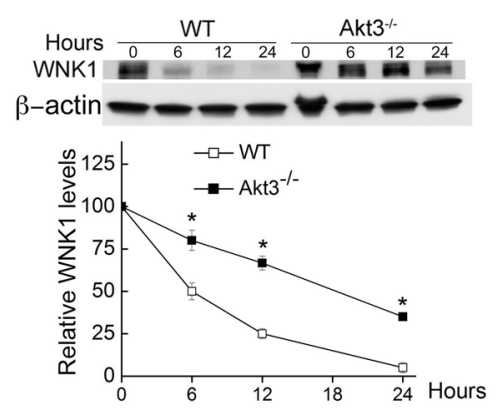

+ WNK1 SiRNA



+ Akt3/WNK1 siRNA



$\mathrm{Akt}^{-/-}$



C: control; Wi:+WNK1 siRNA; Ai:+Akt3siRNA
D



H

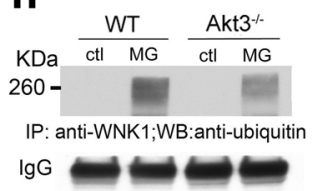

I Human
preadipocytes Control WNK1

P-NDRG1 NDRG1

Akt3 GAPDH



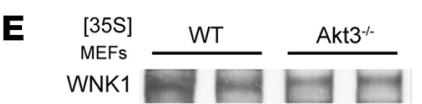

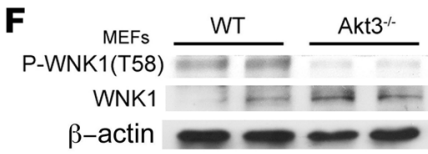

Figure 4. Akt3 regulates SGK1 activity via WNK1. (A) Western blot analysis of WNK1 expression in Akt3 ${ }^{-1-}$ MEF and Akt3 ${ }^{-/-}$adipocytes of female mice; WNK1 and Akt3 expression in 3T3-L1 cells treated with Akt3 siRNA or WNK1 siRNA; WNK1 and phospho-NDRG1/NDRG1 ratio (SGK1 activity) in human preadipocytes treated with Akt3-specific siRNA. $n=3$. (B) Western blot analysis of WNK1, NDRG1, Phospho-NDRG1 expression in Akt3-1- MEF transfected with WNK1 siRNA for 24 hours. $n=4$. (C) Adipogenesis assay using 3T3-L1 cells transfected with Akt3 siRNA or WNK1 siRNA, or cotransfected with Akt3/WNK1 siRNA for 24 hours. Scale bar: 100 $\mu \mathrm{m} . n=5$. (D) Comparable WNK1 mRNA expression in WT and Akt3-l- MEF using quantitative PCR; $\beta$-actin mRNA used as a loading control. $n=7$. (E) Immunoprecipitation of WNK1 from WT and $A k t 3^{-/-}$MEF lysates after a 2-hour pulse-label with [ ${ }^{35} \mathrm{~S}$ ] methionine. Experiment was repeated 3 times. $n=4$. (F) Decreased phosphorylation of WNK1 (Thr58) in Akt3-deficient MEF. $n=4$. (G) WNK1 expression in WT and Akt3 ${ }^{-/-}$MEF treated with cycloheximide (10 $\mu$ g/ml) for 6,12 , and 24 hours; $\beta$-actin used as a loading control. $n=3$. (H) Immunoprecipitation of WNK1 from WT and Akt3 ${ }^{-1-}$ MEF lysates with treatment of MG132 (MG, $5 \mu$ M) for 20 hours, followed by SDS-PACE and immunoblotting using anti-ubiquitin antibody and anti-WNK1 antibody. $n=4$. (I) WNK1 expression in WT and $A k t 3^{-1-}$ MEF treated with proteasome inhibitor MG132 (MG, $5 \mu \mathrm{M}$ ) or Bortezomib (Bor, $10 \mathrm{nM}$ ) for 24 hours; $\beta$-actin used as a loading control. $n=3$. (J) Expression of WNK1, SGK1, NDRG1, and phosphorylation of NDRG1 in WT and Akt1-/- MEF cells. Scale bar: $100 \mu \mathrm{m}$. Data represent means \pm SEM. ${ }^{*} P<0.05$ by 2 -tailed Student's $t$ test.

cally elevated in the nuclei of $A k t 3^{-1-} \mathrm{MEF}$, where FOXO1 is phosphorylated (Figure 3D). Furthermore, we observed increased SGK1 phosphorylation and activity in WAT of $A k t 3^{-1-}$ mice (Figure 3E).

To test whether increased FOXO1 phosphorylation in $A k t 3^{-1-} \mathrm{MEF}$ is SGK1 dependent, we assessed the protein level and phosphorylation of FOXO1 in the presence of a specific SGK1 inhibitor, GSK650394. GSK-650394 induced rapid and pronounced inhibition of FOXO1 phosphorylation in $\mathrm{Akt}^{-1-}$ MEF, while inducing only a moderate and delayed decrease in FOXO1 phosphorylation in WT MEF (Figure 3F). The inhibition of SGK1 by GSK650394 was confirmed by the lack of NDRG1 phosphorylation in treated cells (Figure 3G). Furthermore, GSK650394 strongly inhibited adipogenesis in Akt3 ${ }^{1-} \mathrm{MEF}$ and rendered it similar to that in WT MEF (Figure $3 \mathrm{H}$ ). We observed no significant changes in FOXO1 phosphorylation in $A k t 3^{-1-} \mathrm{MEF}$ treated with specific inhibitors of cyclin-dependent kinase 2 (CDK2), casein kinase 1 (CK1), nor dual-specificity tyrosine phosphorylation-regulated kinase $1 \mathrm{~A}$ (DYRK1A), all of which are kinases that can phosphorylate FOXO1 (Supplemental Figure 1; supplemental material available online with this article; https://doi.org/10.1172/jci.insight.95687DS1).

$A K T 3$ regulates SGK1 activity via WNK1. Akt and SGK share upstream activation pathways. As we observed increased activation of SGK1 in the absence of a similar increase in Akt1 activity, we searched for 
A



B



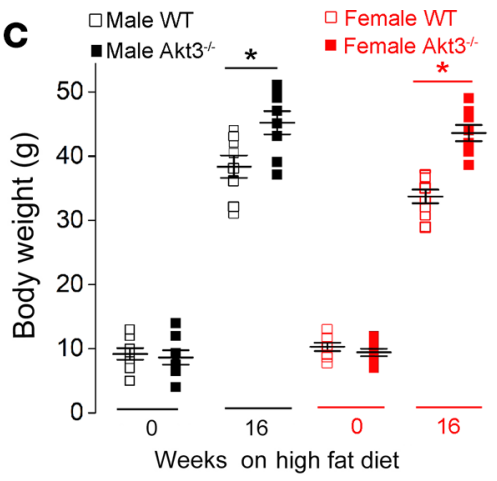

G

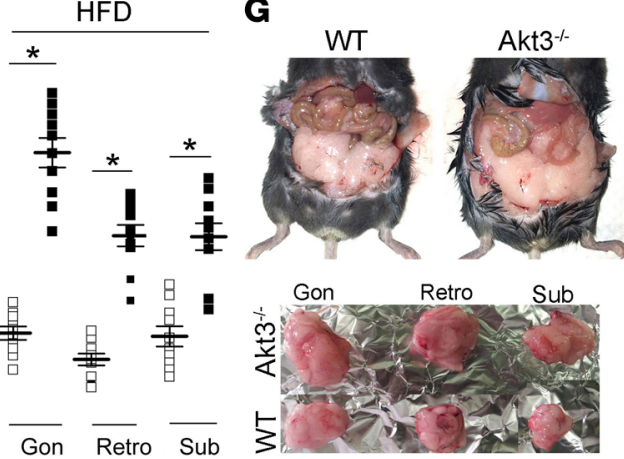

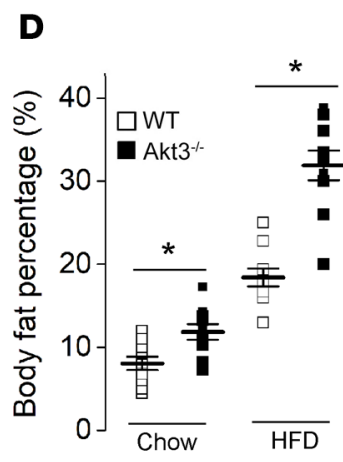

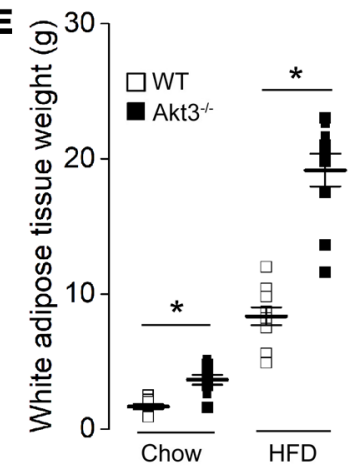

$\mathbf{F}$

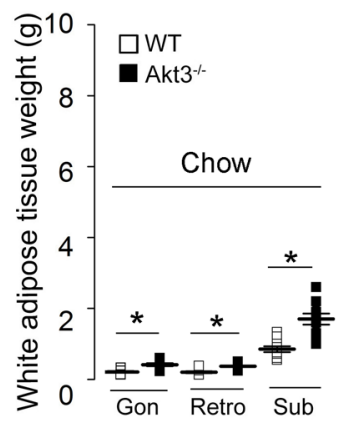

WT

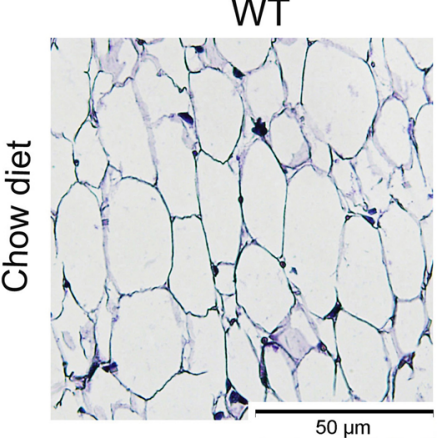

Akt3 $^{-1-}$

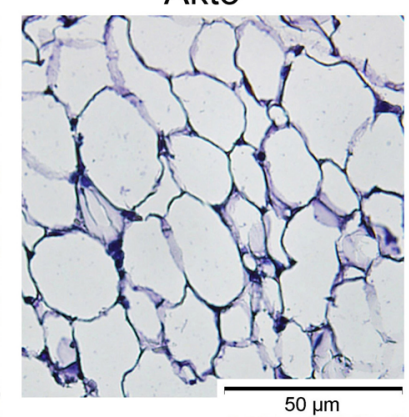

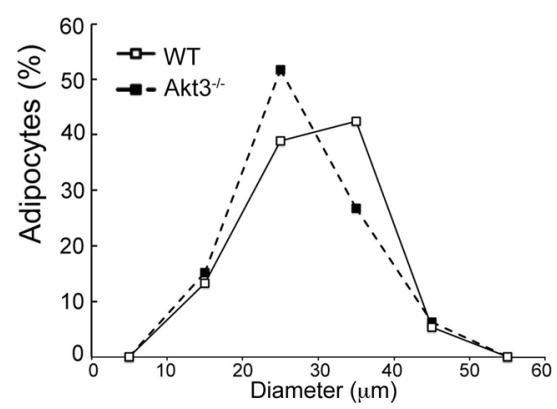

Figure 5. Deficiency of Akt3 promotes white adipose tissue deposition and weight gain in mice. (A) Body weight of sex-matched WT and $A k t 3^{-/-}$mice fed a chow diet at $4,8,10,12,14,16,18,20$, and 22 weeks of age. $n=20$ (10 male, 10 female). (B) Body weight changes in sex-matched WT and $A k t 3^{-1-}$ mice fed an HFD. $n=16$ (8 male, 8 female). (C) Body weight of male and female WT and Akt3 ${ }^{--}$mice fed an HFD for 0 and 16 weeks. $n=8$ (4 male, 4 female). (D)

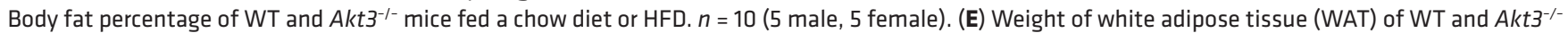
mice on a chow diet or an HFD. $n=10$ (5 male, 5 female). (F) Weight of gonadal (gon), retroperitoneal (retro), and s.c. (sub) white adipose tissue from WT and $A k t 3^{-/-}$mice fed a chow diet or an HFD. $n=10$ (5 male, 5 female). (G) Abdominal fat image of representative 10 -month-old female WT and Akt3-/- mice on a chow diet (upper panel). Lower panel shows gonadal (gon), retroperitoneal (retro), and s.c. (sub) fat pad isolated from WT and $A k t 3^{-/-}$mice on a chow diet. (H) Lean mass was calculated by subtracting the fat mass from body weight of mice fed a chow diet or HFD. $n=10$. (I) H\&E-stained paraffin-embedded sections of gonadal WAT from 8-week-old female WT and Akt3 ${ }^{-1-}$ mice fed a chow diet. Graph to the right shows size distribution of adipocytes. $n=5$. Data represent means \pm SEM. ${ }^{*} P<0.05$ by ANOVA with Bonferroni post-hoc test $(\mathbf{A}$ and $\mathbf{B})$ and 2 -tailed Student's $t$ test (C-H).

alternative pathways that specifically lead to SGK1 activation. Serine/threonine kinase WNK1 promotes SGK1 activation without affecting Akt (29). WNK1 level was enhanced in $A k t 3^{-/}$MEF, in adipocytes isolated from WAT of $A k t 3^{-/-}$mice, as well as in 3T3-L1 cells and in human preadipocytes with Akt3 silenced by Akt3 siRNA (Figure 4A). Silencing of WNK1 significantly inhibited SGK1 activation and adipogenesis in $\mathrm{Akt}^{-/-}$cells but not in control cells, demonstrating that WNK1 is a link between Akt3, SGK1, and adipogenesis (Figure 4, B and C). We next investigated the mechanism of increased WNK1 in Akt3 deficiency. Neither WNK1 protein synthesis nor WNK1 mRNA level were increased in $A k t 3^{-/-} \mathrm{MEF}$ (Figure 4, D and E). Akt3 can regulate protein expression at a posttranslational level (6). WNK1 is a known Akt substrate; however, whether WNK1 protein expression is regulated by Akt phosphorylation and spe- 
A

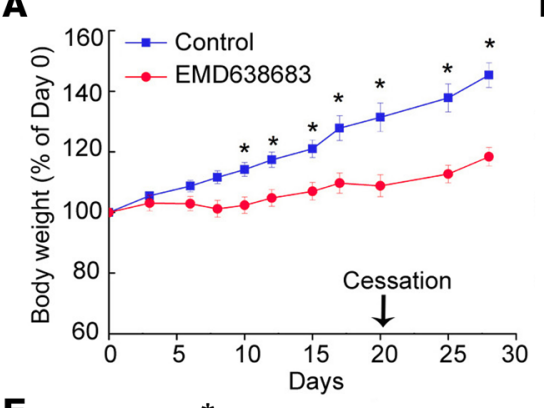

E



$\mathbf{G}$

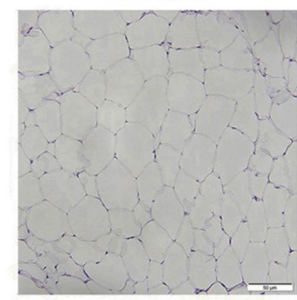

EMD638683
B



C

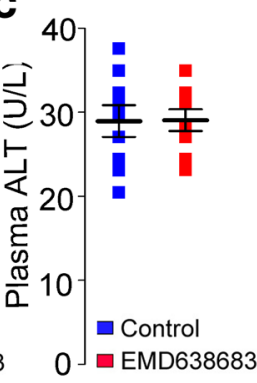



D
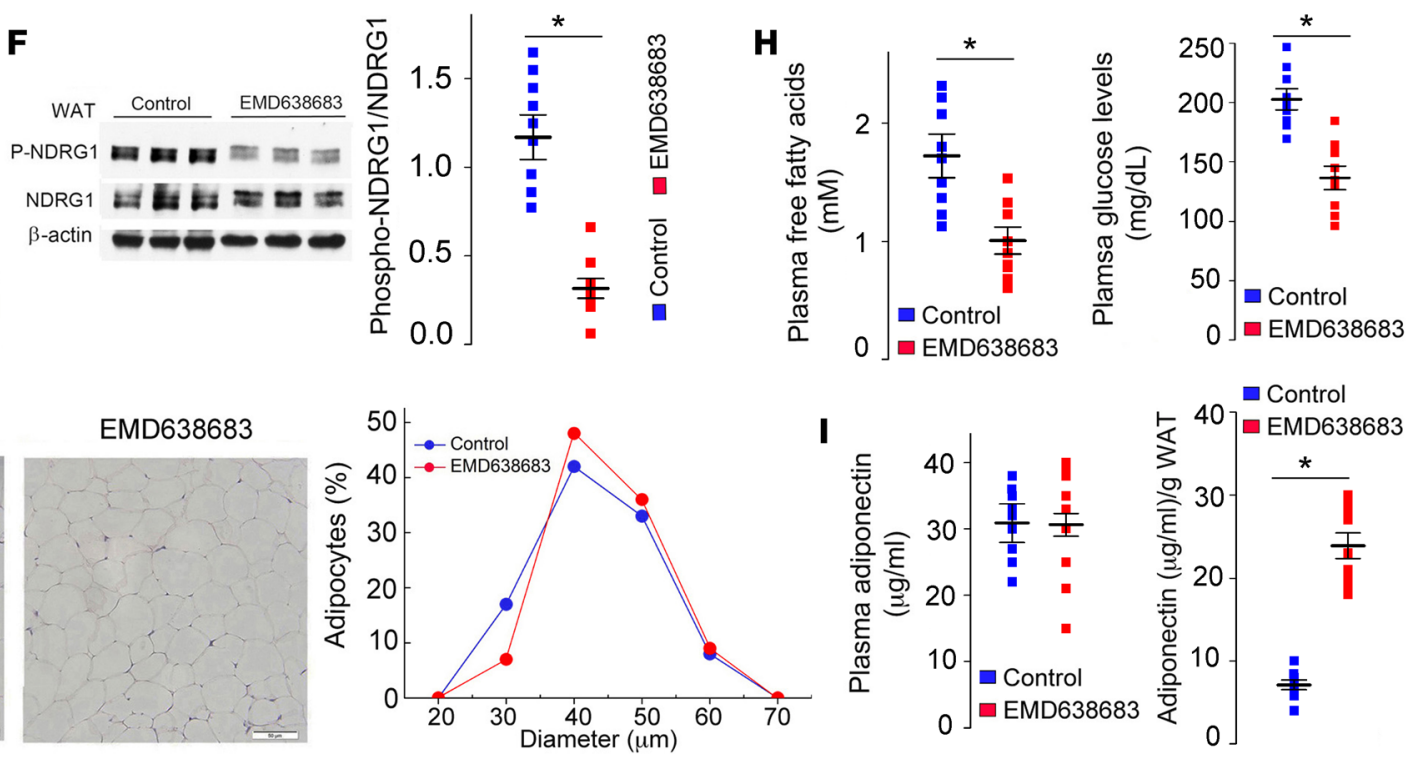

Figure 6. SGK1 inhibitor EMD638683 prevents diet-induced obesity in Akt3 ${ }^{-1-}$ mice. Female $A k t 3^{-/-}$mice fed HFD orally received EMD638683 (EMD mice, $n=9$ ) or DMSO (control, $n=9$ ). (A) Relative body weight (\% of day 0 ) in EMD and control group. Treatment was terminated at day 21. (B) Lean mass of EMD and control mice. (C) Plasma liver transaminases (AST and ALT) in EMD and control mice. (D) Weight of gonadal (gon), retroperitoneal (retro), and s.c. (sub) white adipose tissue from EMD and control mice. (E) Body fat percentage in EMD mice and control mice. (F) Western blotting analyses of phospho-NDRG1 and NDRG1 expression in WAT from EMD mice and control mice. (G) H\&E staining of gonadal WAT from EMD and control mice. Graphs to the right show size distribution of adipocytes. Scale bar: $50 \mu \mathrm{m}$. (H) Plasma free fatty acids level and glucose level in EMD and control mice. (I) Absolute adiponectin and relative adiponectin levels (relative to WAT weight) in EMD mice and control mice. Data represent means $\pm S E M .{ }^{*} P<0.05$ by ANOVA with Bonferroni post-hoc test (A) and 2-tailed Student's $t$ test (B-F, H, I).

cific Akt isoform involved is unknown. Phosphorylation of WNK1 at T58 was significantly decreased in $A k t 3^{-1-}$ MEF (Figure 4F). Furthermore, stability of WNK1 was significantly increased by Akt3 deficiency (Figure 4G), suggesting that WNK1 protein expression is regulated by Akt3 at a posttranslational level. Protein expression of WNK kinases may be regulated via the ubiquitin-proteasome pathway (30). A specific proteasome inhibitor (MG132) induced the accumulation of polyubiquitinated WNK1, which was much more pronounced in WT MEF (Figure 4H), especially when taking into account the lower level of WNK1 in WT cells. Treatment of WT MEF but not $A k t 3^{-1-}$ MEF with proteasome inhibitors resulted in a pronounced increase in WNK1 protein expression (Figure 4I), suggesting that WNK1 is polyubiquitinated in MEF and subsequently degraded through the proteasome pathway. Neither WNK1 expression nor SGK1 activity was changed in Akt1-deficient MEF cells (Figure 4J), demonstrating that Akt3 specifically regulates WNK1 expression and SGK1 activity during adipogenesis.

Akt3 deficiency promotes obesity in mice. We next tested whether increased adipogenesis of $A k t 3^{--} \mathrm{MEF}$ that we observed in vitro is associated with both the accumulation of WAT and increased weight gain in Akt3-1mice. The body weights of $A k 3^{--}$mice and WT mice fed a chow diet were similar (Figure 5A). However, when age- and sex-matched $A k t 3^{--}$and WT mice were fed an HFD, Akt3 deficiency was associated with both increased weight gain (Figure 5B) and body fat percentage (Figure 5D). This difference was more pronounced in female mice (Figure 5C). We compared the weights of white adipose tissue (WAT) from various depots 
of WT and $A k t 3^{--}$mice fed a chow diet or HFD. Combined WAT mass was 2 times higher in $A k t 3^{-1-}$ mice fed a chow diet as compared with WT controls (Figure 5E). WAT were also higher in $A k t 3^{-1-}$ mice on HFD (Figure 5E). We observed a higher expansion of fat pads from various regions in $A k t 3^{-/-}$mice (Figure $5 \mathrm{~F}$ ). $A k t 3^{-1-}$ mice on a chow diet also displayed a universally increased fat mass (2.7- to 3.6-fold increase at different depots) at 10 months of age (Figure 5G). Lean mass of WT and $A k t 3^{-1-}$ mice fed a chow diet or HFD (Figure $5 \mathrm{H}$ ) was similar, confirming that the increased adipose tissue deposition underlies the elevated body weight in $A k t 3^{-1-}$ mice. The mean adipocyte volume was decreased by $5 \%$ in $A k t 3^{--}$mice fed a chow diet as compared with the corresponding WT group (Figure 5I). Considering a 2-fold increase in WAT weight in mice on chow (Figure 5E), we infer that there are at least 1.7 times more adipocytes in $A k 3^{-1-}$ mice, indicating that adipocyte hyperplasia contributes to increased adiposity of these mice.

$\mathrm{Akt}^{-1-}$ mice on HFD manifest hepatic steatosis and impaired glucose homeostasis. Obesity is frequently associated with hepatic steatosis (31) and insulin resistance (32). We observed an increased liver weight (Supplemental Figure 4A) and more severe hepatic steatosis (Supplemental Figure 4B) in $A k t 3^{-/-}$mice fed an HFD. In agreement with previous reports $(31,33)$, we found a negligible expression of Akt 3 in the livers of WT mice (Supplemental Figure 4C), suggesting that the enhanced hepatic steatosis in $A k t 3^{-/-}$mice is secondary to obesity. Expression of PPAR $\gamma$ in livers of WT and $A k t 3^{-1-}$ mice were similar (Supplemental Figure 4D), ruling out a mechanistic role for this transcription factors in enhanced obesity and steatosis in $A k t 3^{--}$mice (32). No significant difference in random blood glucose was observed between WT and $\mathrm{Akt}^{-/-}$littermate controls fed a chow diet (Supplemental Figure 4E). In contrast, $A k t 3^{-1-}$ mice fed an HFD developed hyperglycemia (Supplemental Figure 4E). To investigate systemic insulin sensitivity, we performed insulin tolerance tests (ITT) and glucose tolerance tests (GTT). Both, chow diet-fed WT mice and $A k t 3^{--}$mice responded promptly to an insulin or glucose challenge, and little to no difference between 2 groups was observed (Supplemental Figure 4, F and G). ITT revealed a significantly reduced hypoglycemic response to insulin in HFD-fed $A k t 3^{--}$mice as compared with WT littermates (Supplemental Figure $4 \mathrm{H}$ ). $A k t 3^{-1-}$ mice showed significantly higher glucose levels on a glucose challenge (Supplemental Figure 4I). While insulin levels were comparable between the 2 groups on a chow diet, significantly higher insulin levels were observed in $A k t 3^{-1-}$ mice on an HFD (Supplemental Figure $4 \mathrm{~J}$ ), indicating the development of insulin resistance. These results suggest that the hepatic steatosis and impaired glucose homeostasis in Akt3-deficient mice are secondary to obesity rather than primary changes.

Disturbed energy balance is not the primary mechanism leading to obesity in Akt3 ${ }^{-1-}$ mice. To investigate if the obesity in Akt3-deficient mice was due to disturbed energy balance, we assessed oxygen consumption $\left(\mathrm{VO}_{2}\right)$, the respiratory exchange ratio (RER), heat production, and locomotor activity in WT and $A k t 3^{-/-}$mice. There was no significant difference in heat production in female mice (Supplemental Figure 2A and Supplemental Figure 6C), while an increase in heat production was observed in male $A k t 3^{-1-}$ mice (Supplemental Figure $3 \mathrm{~A}$ and Supplemental Figure $6 \mathrm{~F}$ ). No statistically significant differences in the $\mathrm{VO}_{2}$ (Supplemental Figure $2 \mathrm{~B}$ and Supplemental Figure $6 \mathrm{~A})$, carbon dioxide production $\left(\mathrm{VCO}_{2}\right)($ Supplemental Figure $2 \mathrm{C}$ and Supplemental Figure 6B), RER (Supplemental Figure 2D), horizontal $x$ axis movements (Supplemental Figure 2E), or vertical $z$ axis movements (Supplemental Figure $2 \mathrm{~F}$ ) between female WT and $A k t 3^{-/-}$groups were found. Despite the fact that $A k t 3^{-/-}$males may be slightly less active at the beginning of the dark cycle (Supplemental Figure 3, E and F), they have increased $\mathrm{VO}_{2}$ and $\mathrm{VCO}_{2}$, predominantly during the light cycle as compared with WT male mice (Supplemental Figure 3, B and C, and Supplemental Figure 6, D and E), which might contribute to less prominent weight gain in male $\mathrm{Akt}^{-1-}$ mice as compared with females. No difference in RER between male WT and $A k t 3^{-/-}$groups was observed (Supplemental Figure 3D). In agreement with previously published data, food intake was increased significantly during a 5-day period after the transfer of mice on an HFD; however, we observed no significant difference in food intake between the 2 groups during an initial 5-day period of HFD (Supplemental Figure 2G) nor at 20 weeks of HFD feeding (Supplemental Figure $2 \mathrm{H}$ ). These results strongly suggest that a disturbed energy balance is not the primary mechanism leading to obesity in mice lacking Akt3.

Inhibition of SGK1 prevents diet-induced obesity in Akt3-deficient mice. We next tested whether increased weight gain and fat expansion in $A k t 3^{-/-}$mice fed an HFD is SGK1 dependent. EMD638683 is a small molecule SGK1 inhibitor with good oral bioavailability that has been successfully used to treat hypertension and colonic tumors in mice $(34,35)$. Oral administration of the EMD638683 suppressed increased weight gain in $\mathrm{Akt3}^{-/-}$mice fed an HFD (Figure 6A). Importantly, there was no statistically significant differences in lean mass, liver and heart weights, or tibia length between the treated and control groups (Figure 6B 
and Supplemental Figure 5, A and B), ruling out a significant effect of EMD638683 on animal growth. Food consumption was not affected by the EMD638683 (Supplemental Figure 5C). Plasma levels of liver transaminases alanine aminotransferase (ALT) and aspartate aminotransferase (AST) were not changed by EMD638683, indicating that EMD638683 had no significant hepatotoxicity (Figure 6C). Deposition of WAT was universally decreased by EMD638683 (Figure 6D), and body fat percentage was 2.5-fold lower in treated mice as compared with the control group (Figure 6E). Decreased phosphorylation of NDRG1 confirmed the suppression of SGK1 activity in WAT of treated mice (Figure 6F). Interestingly, EMD638683 treatment resulted in a slight increase in the mean adipocyte volume (Figure 6G). Taking into account a 3.2-fold increase in WAT weight in control mice (Figure 6D), we infer that EMD638683 treatment reduced the number of adipocytes 3.5-fold, supporting our hypothesis that that EMD638683 prevents increased weight gain in $A k t 3^{-1-}$ mice by suppressing adipocyte hyperplasia. EMD638683 treatment also induced normalization of plasma FFA, insulin, and glucose levels (Figure 6H and Supplemental Figure 5D). Despite the similar absolute concentrations of plasma adiponectin in treated mice and control mice, the adiponectin levels per gram of WAT were remarkably higher in treated mice (Figure 6I), demonstrating that EMD638683 improved endocrine function of WAT.

\section{Discussion}

In the current study, we documented the existence and function of Akt3/WNK1/SGK1 signaling pathway in adipocytes in vivo and in vitro. We demonstrated that dysregulation of this pathway predisposes mice to diet-induced obesity and insulin resistance. Mechanistically, we demonstrated that a lack of Akt3 activity in preadipocytes leads to activation of adipogenesis via increased expression of WNK1, associated upregulation of SGK1 activity, and SGK1-mediated downregulation of a critical negative regulator of adipogenesis FOXO1. We further demonstrated that small molecule inhibition of this pathway prevents diet-induced obesity and ameliorates metabolic parameters in mice with Akt3 deficiency.

Three Akt isoforms, encoded by 3 separate genes, are expressed in mammals (8). Akt1 and Akt2 involvement in the regulation of metabolism is well known (36). On the other hand, the role of Akt3 in metabolic disease has not yet been described (37). All 3 Akt isoforms are expressed in adipocytes, and function of the Akt1 and Akt2 in adipocytes has been previously studied. Akt1 expression in primary MEF promotes adipocyte differentiation (9). FOXO1 phosphorylation by Akt1 is considered to be a major mechanism of Akt1 contribution to adipogenesis (9). Akt2, on the other hand, is dispensable for normal development of WAT, yet it is required for diet-induced adipocyte precursor activation and adipogenesis (13). The loss of Akt2 does not significantly impair differentiation of murine embryonic fibroblasts into adipocytes but instead regulates adipocyte apoptosis (11). Transduction of insulin-induced signaling is considered to be a major contribution of Akt2 to adipogenesis (15). It has been shown that Akt1 and Akt2 are required for adipose expansion and maintenance, presumably through the insulin/IGF-1 receptors/Akt signaling pathway (14). The loss of Akt1/2 leads to severe insulin resistance, hepatic steatosis, and hepatomegaly in lean mice (14). In contrast, deficiency of Akt3 leads to these pathological changes in response to an HFD. Neither contribution of Akt3 to metabolic disease nor to adipocyte function has been described before, to our knowledge. Original studies failed to find a role for Akt3 in metabolism in mice (37-39). Our study using Akt3-deficient mice challenged with an HFD establishes a surprising and prominent role of Akt3 in the suppression of development of obesity via regulation of adipogenesis.

Consistent with FOXO1 being a principal negative regulator of adipocyte differentiation $(4,40)$, we observed a strong correlation between adipogenesis and FOXO1 levels in preadipocytes. Overexpression of FOXO1 was sufficient to overcome the phenotype of $A k t 3^{-1-}$ preadipocytes, demonstrating that downregulation of FOXO1 is the principal effect of Akt3 deficiency in adipocytes. We confirmed that FOXO1 is a major target for phosphorylation by Akt1 in WT cells $(9,41)$. However, in $A k t 3^{-/-}$preadipocytes, an alternative kinase was responsible for enhanced FOXO1 phosphorylation. We tested several likely candidates $(25,42)$ and unequivocally identified kinase as SGK1, a serine/threonine kinase that has multiple roles but is mostly known for regulation of ion channels (43). Absence of Akt3 activity does not significantly change the protein level of SGK1 in MEF, but it leads to its activation and nuclear translocation. Similar to Akt1, SGK1 then phosphorylates FOXO1, leading to the nuclear exclusion of FOXO1. Interestingly, we observed increased expression as well as activation of SGK1, particularly in female mice, the sex displaying a particularly strong phenotype in Akt3 deficiency. The mechanism of sex-specific regulation of SGK1 is currently under investigation. While increased SGK1 levels in the WAT of obese 
mice and humans (44) and association of SGK1 polymorphism with metabolic syndrome $(17,45)$ have been reported, our study is the first to our knowledge to demonstrate in vivo that dysregulation of SGK1 promotes adiposity and may lead to obesity.

A lack of increased Akt activation in $A k t 3^{-/-}$cells prompted us to focus on WNK1, a kinase known to activate SGK1 but not Akt (29). WNK1, mostly known for regulation of the renal tubular salt and potassium transport (46), has poorly understood relationship with Akt and SGK1. Increased expression of WNK1 in cells is sufficient to promote activation of SGK1 (18). WNK1 forms a complex with SGK1 and promotes SGK1 activation by increasing phosphorylation of Thr-256 in the SGK1 activation loop $(18,29)$. WNK1 does not directly phosphorylate SGK1, and binding of WNK1 to SGK1 is not sufficient for its activation (47). WNK1 is also a substrate for Akt and SGK1. Both kinases can phosphorylate WNK1 at Thr58/60; however, the role of this phosphorylation in WNK1 function has not been established. While in vitro studies have demonstrated that Akt1 can phosphorylate WNK1, neither the specific Akt isoform that phosphorylates WNK1 in cells in vivo nor the physiological role of this phosphorylation has been established. Our studies clearly demonstrate that, in adipocytes, WNK1 is preferentially phosphorylated by Akt3. Furthermore, we demonstrated that phosphorylation leads to WNK1 polyubiquitination and degradation via ubiquitin-proteasome pathway, establishing a mechanistic link between Akt3 and SGK1 activity. Our study is the first to demonstrate that WNK1 can contribute to obesity and may represent a direct link between obesity and hypertension.

We demonstrated that pharmacological inhibition of SGK1 prevents diet-induced obesity, particularly in young $\mathrm{Akt}^{-3^{--}}$animals. The number of adipocytes, but not adipocyte volume, was reduced by EMD638683, strongly suggesting that EMD638683 prevents obesity through inhibition of adipogenesis, in agreement with in vitro data. Moreover, we found that metabolic parameters such as FFA, insulin, and glucose levels were improved by the EMD638683. Adipocyte hyperplasia is particularly important in childhood-onset obesity. Moreover, it strongly predisposes for later obesity. Obesity, hypertension, and insulin resistance defined as pediatric metabolic syndrome have become increasingly common. Our findings along with known antihypertensive effect (34) suggest that pharmacological regulation of SGK1 may be an approach for the treatment of childhood obesity and pediatric metabolic syndrome.

Since this work was performed in a global $\mathrm{KO}$ of $\mathrm{Akt} 3$, we cannot completely exclude the contribution of Akt3 expression in nonadipose issues to the described phenotype in $A k t 3^{-1-}$ mice. For example, Akt3 is highly expressed in the brain, and its deficiency leads to a number of cognitive and behavioral derangements in mice (48-51). While we cannot exclude contribution of behavioral changes to increased adiposity in $A k t 3^{-1-}$ mice, we ruled out changes in food intake, energy expenditure, and physical activity as possible contributors to this phenotype. Another example is potential contribution of adipose tissue macrophages to increased adiposity in $A k t 3^{-1-}$ mice. It should be noted, though, that while $A k t 3^{-1-}$ macrophages display increased pinocytosis and accumulation of lipoprotein-derived cholesterol $(6,52)$, it remains unclear whether this macrophage phenotype contributes to obesity in $A k t 3^{-1-}$ mice. Despite these theoretical possibilities, our multiple in vitro experiments reproduced in various primary cells and cell lines demonstrate clearly the increased adipogenic potential of $\mathrm{Akt}^{-/-}$preadipocytes in the absence of contributions of other factors. These results support the mechanism of the diet-induced obesity that we proposed.

Taken together, our data suggests that interplay between Akt3, WNK1, and SGK1 regulates adipogenesis in vivo and that dysregulation of this pathway can lead to increased adipogenesis and obesity and insulin resistance.

\section{Methods}

Materials. Antibodies against Akt1 (catalog 75692S), Akt2 (catalog 2964S), pan-Akt (catalog 4691S), phospho-Akt (Ser473; catalog 4060S), phospho-Akt (Thr308; catalog 13038S), FOXO1 (catalog 2880S), phospho-FOXO1 (Ser256; catalog 9461S), PPAR $\gamma$ (catalog 2435S), adiponectin (catalog 2789S), NDRG1 (catalog 5196S), phospho-NDRG1 (catalog 5482S), lamin B1 (catalog 12586S), FABP4 (catalog 2120S), $\mathrm{C} / \mathrm{EBP} \alpha$ (catalog 2295S), HSL (catalog 4107S), phospho-HSL (Ser563; catalog 4139S), ATGL (catalog 2439S), and FAS (catalog 3189S) were purchased from Cell Signaling Technology. Antibody against SGK1 was purchased from Biovision (catalog 3459-100); antibodies against phospho-SGK1 (Ser422) (sc16745), and Akt3 (sc11521) were from Santa Cruz Biotechnology. Antibody against WNK1 was from Genetex (GTX106197). Anti-MGL antibody (catalog 100035), mouse adiponectin EIA Kit, and Glucose Colorimetric Assay Kit were from Cayman; the Serum/Plasma Fatty Acid Detection Kit was from Zen-Bio; the Ultra Sensitive Mouse Insulin ELISA Kit was from Crystal Chem. Control siRNA, Akt1 siRNA, and Akt3 
siRNA were purchased from Santa Cruz Biotechnology Inc. Lipofectamine RNAi ${ }_{\text {MAX }}$, BODIPY 493/503, and LipidTOX Red Neutral Lipid Stain were from Invitrogen; and Fugene HD was from Promega. pcDNA3.1-HA-Akt3 was a gift from Morris Birnbaum (University of Pennsylvania, Philadelphia, PA, USA); the Akt3 mutant pcDNA3.1-HA- Akt3 ${ }^{\text {nmf350 }}$ was a gift from Wayne N. Frankel (the Jackson Laboratory); and pCMV6-human FOXO1 were from Origene (SC118890). Human s.c. white preadipocytes were from PromoCell. SGK1 inhibitor (GSK650394), CDk2 inhibitor (sc221409), CK1 inhibitor (D4476), and proteasome inhibitors MG132 and Bortezomib were from Santa Cruz Biotechnology Inc. DYRK1A inhibitor (Harmine) and IKK inhibitor (BAY11-7082) were from Cayman Chemical.

Animal procedures. We used littermate-derived, sex-, age-, and genetic background-matched mice in our experiments. $A k t 3^{3^{--}}, \mathrm{Akt}^{2^{--}}$, and $A k t 1^{-/-}$mice were generated by Nissim Hay's lab (Department of Molecular Genetics, College of Medicine, University of Illinois at Chicago, Chicago, Illinois, USA). Akt ${ }^{3^{--}}$mice were backcrossed $\geq 10 \times$ to $\mathrm{C} 57 \mathrm{Bl} / 6$. After the last backcross, heterozygotes were mated to create background matched WT and $A k t 3^{--}$mice for the present studies. For the diet-induced obesity study, 4-weekold mice were fed an HFD (TD88137, Harlan Teklad, 42\% of calories from fat).

For the treatment of HFD-fed mice with SGK1 inhibitor, 4- to 6-week-old Akt3-deficient mice were randomized into 2 groups (control and EMD group) and both were fed an HFD. EMD group mice received EMD638683 (dissolved in DMSO, $600 \mathrm{mg} / \mathrm{kg} /$ day) orally, and control mice received an equivalent amount of DMSO daily. Body weights were taken every 2-3 days for 21 days. Mice were sacrificed 7 days (day 28) after cessation of EMD638683/DMSO administration. Tibia lengths were measured, and blood and fat tissues were collected for further analysis.

GTT and ITT. Mice were fasted for 4 hours, and then a 0.6 unit of insulin per $\mathrm{kg}$ of body weight was administered by i.p. injection. Whole-blood glucose was determined at $0,15,30,45$, and 60 minutes. For a GTT, the mice were fasted overnight, and glucose was administered i.p. ( $2 \mathrm{~g} / \mathrm{kg}$ body weight). Wholeblood glucose was determined at $0,15,30,45,60$ and 120 minutes. The data on GTT and ITT were both presented as the relative percentage of the initial levels.

Plasma insulin, free fatty acids (FFAs), and adiponectin. Mice were fasted overnight for 10 hours before blood collection for the analysis of insulin, FFAs, and adiponection. Plasma insulin and FFA levels were measured using an Ultra Sensitive Mouse Insulin ELISA Kit (Crystal Chem) and a Serum/Plasma Fatty Acid Detection Kit (Zen-Bio). Plasma adiponectin levels were assessed using an Adiponectin ELISA kit (Cayman).

In vitro adipogenesis assay. Confluent 3T3-L1 preadipocytes were cultured in differentiation media containing $2.5 \mu \mathrm{g} / \mathrm{ml}$ insulin (Sigma Aldrich), $50 \mu \mathrm{M}$ dexamethasone (Cayman Chemical), and $500 \mu \mathrm{M}$ 3-isobutyl-1-methylxanthine (IBMX, Cayman Chemical) for 3 days. Then, cells were cultured in maintenance media containing $2.5 \mu \mathrm{g} / \mathrm{ml}$ insulin and $50 \mu \mathrm{M}$ dexamethasone for 5-7 days. Confluent MEF were incubated with differentiation media containing $5 \mu \mathrm{g} / \mathrm{ml}$ insulin, $1 \mu \mathrm{M}$ dexamethasone, and $500 \mu \mathrm{M}$ IBMX for 3 days, and then for an additional 4 days in the maintenance media. After differentiation, cells were fixed with $4 \%$ formaldehyde and stained with Oil red O. Human s.c. white preadipocytes were cultured in human preadipocyte growth media. After reaching confluence, cells were treated with preadipocyte differentiation medium for in vitro adipogenesis assay.

Isolation of cytoplasmic and nuclear proteins. WT and $A k t 3^{-1-} \mathrm{MEF}$ of the same passage were synchronized by overnight serum starvation and cultured in DMEM/20\% FBS media for 24 hours. Then, cells were collected and washed by PBS. Cytoplasmic and nuclear proteins were extracted using the CHEMICON Nuclear Extraction Kit (MilliporeSigma). Protein concentration was determined by BCA kit (Pierce Biotechnology).

Energy metabolism studies. Food intake, energy expenditure, and physical activity were assessed using the Comprehensive Laboratory Animal Monitoring System (CLAMS; Columbus Instruments). For indirect calorimetry studies, 8-week-old WT and $A k t 3^{-1-}$ mice ( $n=6,3$ male, 3 female) from chow diet groups were placed in CLAMS cages and allowed to equilibrate to metabolic cage environments at room temperature $\left(22^{\circ} \mathrm{C}\right)$ for 72 hours before 24 hours of continuous data collection. $\mathrm{VO}_{2}, \mathrm{VCO}_{2}$, heat, RER, and activity were constantly monitored. All measurements were normalized to body weight or lean mass, which did not differ between WT and $\mathrm{Akt}^{3^{--}}$mice for the same sex at onset of the study.

Mouse WAT isolation. WAT were isolated as described (53). Specific definitions of dissected depots are as follows: gonadal WAT, prominent bilateral intraabdominal visceral depots attached to the epididymides in male mice or the ovaries in female mice; retroperitoneal WAT, bilateral depots in abdominal cavity behind the peritoneum on the dorsal side of the kidney; and s.c. WAT, bilateral superficial s.c. WAT between the 
skin and muscle fascia posterior to the lower segment of the upper limbs as well as bilateral superficial s.c. WAT depots between the skin and muscle fascia just anterior to the lower segment of the hind limbs.

Histological analysis. Sections of $10 \mu \mathrm{m}$ for gonadal WAT or brown adipose tissue were cut and mounted on slides. The adipose tissues were stained with H\&E. We determined the distribution of adipocyte size using ImagePro software (Media Cybernetics) to measure the cross-sectional area of at least 100 adipocytes randomly selected from 3 independent histological sections. Five mice of each genotype were used for adipocyte size calculation.

$M E F$ isolation. MEF were isolated from 13.5-day postcoital mouse embryos of WT, $A k t 1^{-/-}, A k t 2^{-/-}$, and $A k t 3^{-/-}$mice as described (54). MEF within 4 passages were used for present experiments.

SiRNA or cDNA transfection. 3T3-L1 cells were transfected with control siRNA, Akt1 siRNA, Akt3 siRNA, Akt3 cDNA, mutant Akt3 cDNA $\left(A k t 3^{\text {nmf350 }}\right.$ ), or FOXO1 cDNA using either Lipofectamine $\mathrm{RNAi}_{\mathrm{MAX}}$ or Fugene HD for 24 hours. After transfection, cells were collected for Western blotting analysis or treated with differentiation medium for adipogenesis assay. Differentiated 3T3 adipocytes were stained with Oil red O or fluorescent markers of neutral lipid such as BODIPY 493/503 (Invitrogen) and LipidTOX Red Neutral Lipid Stain (Invitrogen). $\mathrm{Akt}^{3^{-1}} \mathrm{MEF}$ were transfected with plasmid encoding murine Akt3 gene (hemagglutinin [HA] tagged) and human FOXO1 gene. Twenty-four hours after transfection, cells were either used for assessment of transfection efficiency by Western blotting analysis or used for in vitro adipogenesis assay as described above.

Isolation of adipocytes from WAT. Adipocytes were isolated from gonadal WAT of female WT and Akt $3^{-1-}$ mice fed a chow diet according to the published study with minor modification (55). Briefly, gonadal WAT was washed and minced in adipocyte isolation buffer (100 mM HEPES, $120 \mathrm{mM} \mathrm{NaCl}, 50 \mathrm{mM} \mathrm{KCl}, 5$ $\mathrm{mM}$ glucose, $1 \mathrm{mM} \mathrm{CaCl} 2,1.5 \% \mathrm{BSA}$ ) and then was incubated with the same buffer containing collagenase on a shaking platform for 45 minutes at $37^{\circ} \mathrm{C}$. The resulting mixture was passed through a nylon filter to remove undigested material. The filtrate was centrifuged at $100 \mathrm{~g}$ for 10 minutes at $4^{\circ} \mathrm{C}$. Adipocytes in the upper layer were separated from pelleted nonadipocytes; then, the adipocyte fraction was washed twice in adipocyte isolation buffer using centrifugation at $200 \mathrm{~g}$. The adipocyte and nonadipocyte fraction were then stored in $-80^{\circ} \mathrm{C}$ for future use.

Western blotting analysis. WT and $\mathrm{Akt}^{3^{-1-}} \mathrm{MEF}$ of the same passage were synchronized, collected, and lysed. Equal amounts of lysate protein were separated by SDS-PAGE, transferred onto polyvinylidene difluoride membranes, and incubated with the corresponding antibodies. The specific signals were visualized with ECL plus (GE Healthcare).

In a separate experiment, WT and $A k t 3^{-1-} \mathrm{MEF}$ were differentiated into adipocytes. Cells were treated with the following inhibitors: SGK1 inhibitor (GSK650394; $25 \mu \mathrm{g} / \mathrm{ml})$, CDK2 inhibitor II (1 $\mu \mathrm{M}), \mathrm{CK} 1$ inhibitor (D4476, $50 \mu \mathrm{M}$ ), DYRKA1A inhibitor (Harmine, $1 \mu \mathrm{M}$ ), and IKK inhibitor (BAY 11-7082, 10 $\mu \mathrm{M}$ ), and the levels of FOXO1, phospo-FOXO1, adiponectin, and GAPDH (Sigma Aldrich; G8795) in these cells were assessed by Western blot analysis.

For the WNK1 protein stability analysis, WT and $A k t 3^{-/-}$MEF were treated with cycloheximide $(10 \mu \mathrm{g} /$ $\mathrm{ml}$ ) for 6, 12, and 24 hours. Cells were lysed, and WNK1 was detected by Western blotting. To detect effect of proteasome inhibitors on WNK1, WT and $A k t 3^{-1-}$ MEF were incubated with MG132 (5 $\left.\mu \mathrm{M}\right)$ or Bortezomib (10 nM) for 24 hours. The level of WNK1 and $\beta$-actin (loading control) in cell lysates was assessed by Western blot analysis. To assess the ubiquitination status of WNK1 in WT and $A k t 3^{-1-} \mathrm{MEF}$, cells were incubated with or without $5 \mu \mathrm{M}$ MG132 for 20 hours. Cells were lysed, and WNK1 was immunoprecipitated and detected by immunoblotting using anti-ubiquitin (Santa Cruz Biotechnology; sc271289) and antiWNK1 (Genetex; GTX106197) antibodies.

$q P C R$. For real-time PCR, we homogenized entire adipose tissue or MEF in Trizol (Invitrogen). Then, total RNA was purified from the homogenates. We determined relative mRNA levels on a MyiQ2 Two Color Real-Time PCR Detection System (Bio-Rad) using SYBR Green Supermix (Bio-Rad). The probes were used as follows: SGK1 forward: 5' TTC TGT GGC ACG CCT GAG TAT CTG 3', reverse: 5' GAG GTG CCT TGC CGA GTT TGT AAT 3'; WNK1 forward: 5' CTG AGG GTG ACC AGG GAT TG 3', reverse: 5' TGT GGC ATG CAA GAT ACA GC 3'; GAPDH forward: 5' CCC AGC AAG GAC ACT GAG CAA GAG 3', reverse: 5' CCC AGC AAG GAC ACT GAG CAA GAG 3'.

$\left.{ }^{35} \mathrm{~S}\right]$ methionine labeling. MEF were cultured in the presence of $10 \mu \mathrm{Ci} / \mathrm{ml}\left[{ }^{35} \mathrm{~S}\right]$ Methionine for 2 hours in methionine-free DMEM medium. Then, cells were lysed and WNK1 was immunoprecipitated with anti-WNK1 antibody. Immunoprecipitates were separated by SDS-PAGE and proteins detected by autoradiography. 
Statistics. Data are presented as mean \pm SEM. When 2 groups were compared, the Student's $t$ test or Mann-Whitney $U$ test was used $(P<0.05$ was considered significant). When multiple comparisons were made, a Bonferroni correction was made for each test.

Study approval. We performed all procedures according to protocols approved by the Cleveland Clinic IACUC.

\section{Author contributions}

LD and LZ performed the in vitro and in vivo experimental work. SB performed protein stability analysis and qPCR. RCS performed the operation of CLAMS and data collection/analysis. EP directed the project. TB and JMB critically contribution to the study design. LD and EP designed the study, analyzed the data, and wrote the manuscript.

\section{Acknowledgments}

We thank Maria Febbraio for her advice in insulin resistance studies and Nancy Fiordalisi and Emelye Crehore for proofreading the manuscript. We thank Michael Kim from Case Western Reserve University for his technical assistance in Western blotting analyses. We thank Jessica Altemus for her assistance in insulin-glucose tolerance tests. We also thank Norbert Beier and Markus Klein from Merck KGaA for providing EMD638683. This work was supported in part by NIH grants HL126738 and HL077213 to EAP; 5P01HL073311 to EAP and TVB; HL071625 to TVB; and R01HL122283 and P50AA024333 to $\mathrm{JMB}$ and by American Heart Association postdoctoral fellowship grants 15POST12535000 to RCS and 13POST17240041 to LD.

Address correspondence to: Eugene A. Podrez, Cleveland Clinic, Lerner Research Institute, Department of Molecular Cardiology, 9500 Euclid Avenue, NB5-87, Cleveland, Ohio 44195, USA. Phone: 216.444.1019; Email: podreze@ccf.org.

1. Whitaker RC, Pepe MS, Wright JA, Seidel KD, Dietz WH. Early adiposity rebound and the risk of adult obesity. Pediatrics. 1998;101(3):E5

2. Rosen ED, MacDougald OA. Adipocyte differentiation from the inside out. Nat Rev Mol Cell Biol. 2006;7(12):885-896

3. Cristancho AG, Lazar MA. Forming functional fat: a growing understanding of adipocyte differentiation. Nat Rev Mol Cell Biol. 2011;12(11):722-734.

4. Nakae J, Kitamura T, Kitamura Y, Biggs WH, Arden KC, Accili D. The forkhead transcription factor Foxo1 regulates adipocyte differentiation. Dev Cell. 2003;4(1):119-129.

5. Manning BD, Cantley LC. AKT/PKB signaling: navigating downstream. Cell. 2007;129(7):1261-1274.

6. Ding L, et al. Akt3 deficiency in macrophages promotes foam cell formation and atherosclerosis in mice. Cell Metab. 2012;15(6):861-872.

7. Phung TL, et al. Akt1 and akt3 exert opposing roles in the regulation of vascular tumor growth. Cancer Res. 2015;75(1):40-50.

8. Humphrey SJ, et al. Dynamic adipocyte phosphoproteome reveals that Akt directly regulates mTORC2. Cell Metab. 2013;17(6):1009-1020.

9. Yun SJ, Kim EK, Tucker DF, Kim CD, Birnbaum MJ, Bae SS. Isoform-specific regulation of adipocyte differentiation by Akt/ protein kinase Balpha. Biochem Biophys Res Commun. 2008;371(1):138-143.

10. Fischer-Posovszky P, Tews D, Horenburg S, Debatin KM, Wabitsch M. Differential function of Akt1 and Akt2 in human adipocytes. Mol Cell Endocrinol. 2012;358(1):135-143.

11. Peng XD, et al. Dwarfism, impaired skin development, skeletal muscle atrophy, delayed bone development, and impeded adipogenesis in mice lacking Akt1 and Akt2. Genes Dev. 2003;17(11):1352-1365.

12. Garofalo RS, et al. Severe diabetes, age-dependent loss of adipose tissue, and mild growth deficiency in mice lacking Akt2/PKB beta. J Clin Invest. 2003;112(2):197-208.

13. Jeffery E, Church CD, Holtrup B, Colman L, Rodeheffer MS. Rapid depot-specific activation of adipocyte precursor cells at the onset of obesity. Nat Cell Biol. 2015;17(4):376-385.

14. Shearin AL, Monks BR, Seale P, Birnbaum MJ. Lack of AKT in adipocytes causes severe lipodystrophy. Mol Metab. 2016;5(7):472-479.

15. Gonzalez E, McGraw TE. Insulin-modulated Akt subcellular localization determines Akt isoform-specific signaling. Proc Natl Acad Sci USA. 2009;106(17):7004-7009.

16. Lang F, Böhmer C, Palmada M, Seebohm G, Strutz-Seebohm N, Vallon V. (Patho)physiological significance of the serum- and glucocorticoid-inducible kinase isoforms. Physiol Rev. 2006;86(4):1151-1178.

17. Lang F, Stournaras C. Serum and glucocorticoid inducible kinase, metabolic syndrome, inflammation, and tumor growth. Hormones (Athens). 2013;12(2):160-171.

18. Xu BE, Stippec S, Lazrak A, Huang CL, Cobb MH. WNK1 activates SGK1 by a phosphatidylinositol 3-kinase-dependent and non-catalytic mechanism. J Biol Chem. 2005;280(40):34218-34223.

19. Tokuda S, et al. A novel Akt3 mutation associated with enhanced kinase activity and seizure susceptibility in mice. Hum Mol 
Genet. 2011;20(5):988-999.

20. Rosen ED, et al. C/EBPalpha induces adipogenesis through PPARgamma: a unified pathway. Genes Dev. 2002;16(1):22-26

21. Tan SX, et al. Selective insulin resistance in adipocytes. J Biol Chem. 2015;290(18):11337-11348.

22. Armoni M, et al. FOXO1 represses peroxisome proliferator-activated receptor-gamma1 and -gamma2 gene promoters in primary adipocytes. A novel paradigm to increase insulin sensitivity. J Biol Chem. 2006;281(29):19881-19891.

23. Qiao L, Shao J. SIRT1 regulates adiponectin gene expression through Foxo1-C/enhancer-binding protein alpha transcriptional complex. J Biol Chem. 2006;281(52):39915-39924.

24. Farmer SR. The forkhead transcription factor Foxo1: a possible link between obesity and insulin resistance. Mol Cell. 2003;11(1):6-8.

25. Di Pietro N, et al. Serum- and glucocorticoid-inducible kinase 1 (SGK1) regulates adipocyte differentiation via forkhead box O1. Mol Endocrinol. 2010;24(2):370-380.

26. Zhao Y, Wang Y, Zhu WG. Applications of post-translational modifications of FoxO family proteins in biological functions. J Mol Cell Biol. 2011;3(5):276-282.

27. Greer EL, Brunet A. FOXO transcription factors at the interface between longevity and tumor suppression. Oncogene. $2005 ; 24(50): 7410-7425$.

28. Murray JT, et al. Exploitation of KESTREL to identify NDRG family members as physiological substrates for SGK1 and GSK3. Biochem J. 2004;384(Pt 3):477-488.

29. Xu BE, et al. WNK1 activates SGK1 to regulate the epithelial sodium channel. Proc Natl Acad Sci USA. 2005;102(29):10315-10320.

30. Takahashi D, et al. KLHL2 interacts with and ubiquitinates WNK kinases. Biochem Biophys Res Commun. 2013;437(3):457-462

31. Dummler B, Tschopp O, Hynx D, Yang ZZ, Dirnhofer S, Hemmings BA. Life with a single isoform of Akt: mice lacking Akt2 and Akt3 are viable but display impaired glucose homeostasis and growth deficiencies. Mol Cell Biol. 2006;26(21):8042-8051.

32. Browning JD, Horton JD. Molecular mediators of hepatic steatosis and liver injury. J Clin Invest. 2004;114(2):147-152.

33. Stokes MP, et al. PTMScan direct: identification and quantification of peptides from critical signaling proteins by immunoaffinity enrichment coupled with LC-MS/MS. Mol Cell Proteomics. 2012;11(5):187-201.

34. Ackermann TF, Boini KM, Beier N, Scholz W, Fuchss T, Lang F. EMD638683, a novel SGK inhibitor with antihypertensive potency. Cell Physiol Biochem. 2011;28(1):137-146.

35. Towhid ST, et al. Inhibition of colonic tumor growth by the selective SGK inhibitor EMD638683. Cell Physiol Biochem. 2013;32(4):838-848.

36. Whiteman EL, Cho H, Birnbaum MJ. Role of Akt/protein kinase B in metabolism. Trends Endocrinol Metab. 2002;13(10):444-451

37. Tschopp O, et al. Essential role of protein kinase B gamma (PKB gamma/Akt3) in postnatal brain development but not in glucose homeostasis. Development. 2005;132(13):2943-2954.

38. Cho H, et al. Insulin resistance and a diabetes mellitus-like syndrome in mice lacking the protein kinase Akt2 (PKB beta). Science. 2001;292(5522):1728-1731.

39. George S, et al. A family with severe insulin resistance and diabetes due to a mutation in AKT2. Science. 2004;304(5675):1325-1328.

40. Kim JJ, Li P, Huntley J, Chang JP, Arden KC, Olefsky JM. FoxO1 haploinsufficiency protects against high-fat diet-induced insulin resistance with enhanced peroxisome proliferator-activated receptor gamma activation in adipose tissue. Diabetes. 2009;58(6):1275-1282.

41. Brunet A, et al. Akt promotes cell survival by phosphorylating and inhibiting a Forkhead transcription factor. Cell. 1999;96(6):857-868

42. Tzivion G, Dobson M, Ramakrishnan G. FoxO transcription factors; Regulation by AKT and 14-3-3 proteins. Biochim Biophys Acta. 2011;1813(11):1938-1945.

43. Wulff $\mathrm{P}$, et al. Impaired renal $\mathrm{Na}(+)$ retention in the sgk1-knockout mouse. J Clin Invest. 2002;110(9):1263-1268

44. Li P, Pan F, Hao Y, Feng W, Song H, Zhu D. SGK1 is regulated by metabolic-related factors in 3T3-L1 adipocytes and overexpressed in the adipose tissue of subjects with obesity and diabetes. Diabetes Res Clin Pract. 2013;102(1):35-42.

45. Roth JL, Mobarhan S, Clohisy M. The Metabolic Syndrome: where are we and where do we go? Nutr Rev. 2002;60(10 Pt $1): 335-337$

46. Vidal-Petiot E, et al. WNK1-related Familial Hyperkalemic Hypertension results from an increased expression of L-WNK1 specifically in the distal nephron. Proc Natl Acad Sci USA. 2013;110(35):14366-14371.

47. Heise CJ, et al. Serum and glucocorticoid-induced kinase (SGK) 1 and the epithelial sodium channel are regulated by multiple with no lysine (WNK) family members. J Biol Chem. 2010;285(33):25161-25167.

48. Easton RM, et al. Role for Akt3/protein kinase Bgamma in attainment of normal brain size. Mol Cell Biol. 2005;25(5):1869-1878

49. Howell KR, Floyd K, Law AJ. PKB $/$ AKT3 loss-of-function causes learning and memory deficits and deregulation of AKT/ mTORC2 signaling: Relevance for schizophrenia. PLoS One. 2017;12(5):e0175993

50. Bergeron Y, Bureau G, Laurier-Laurin MÉ, Asselin E, Massicotte G, Cyr M. Genetic Deletion of Akt3 Induces an Endophenotype Reminiscent of Psychiatric Manifestations in Mice. Front Mol Neurosci. 2017;10:102.

51. Wang H, et al. Impaired Spatial Learning is Associated with Disrupted Integrity of the White Matter in Akt3 Knockout Mice. CNS Neurosci Ther. 2017;23(1):99-102.

52. Ding L, Zhang L, Kim M, Byzova T, Podrez E. Akt3 kinase suppresses pinocytosis of low-density lipoprotein by macrophages via a novel WNK/SGK1/Cdc42 protein pathway. J Biol Chem. 2017;292(22):9283-9293.

53. Tran TT, Kahn CR. Transplantation of adipose tissue and stem cells: role in metabolism and disease. Nat Rev Endocrinol. 2010;6(4):195-213.

54. Chua KF, et al. Mammalian SIRT1 limits replicative life span in response to chronic genotoxic stress. Cell Metab. 2005;2(1):67-76.

55. Hosooka T, et al. Dok1 mediates high-fat diet-induced adipocyte hypertrophy and obesity through modulation of PPAR-gamma phosphorylation. Nat Med. 2008;14(2):188-193. 